\title{
Techniques for excision RFI on RATAN-600 radio telescope in $\mathrm{dm}$ ranges
}

\section{Denis Kratov*}

Special Astrophsical observatory 369167,Nizhnij Arkhyz, Russia

E-mail: krat@sao.ru

\section{A.B.Berlin}

Special Astrophisical observatory, Saint Petersburg Branch 196140,Saint Petersburg, Russia

E-mail: abb_36@mail.ru

\section{N.A.Nizhel'skij}

Special Astrophisical observatory 369167,Nizhnij Arkhyz, Russia

E-mail:nizh@sao.ru

\section{P.G. Tsybulev}

Special Astrophisical observatory 369167,Nizhnij Arkhyz, Russia

E-mail: peter@sao.ru

\section{M.G.Mingaliev}

Special Astrophisical observatory 369167,Nizhnij Arkhyz, Russia

E-mail:marat@sao.ru

\section{R.Y.Udovitskiy}

Special Astrophisical observatory 369167,Nizhnij Arkhyz, Russia

E-mail: nemiroff@sao.ru

During several recent years the interference situation at RATAN-600 deteriorated sharply. The analysis of electromagnetic interference at the RATAN-600 radio telescope is presented. The practical experience of radio frequency interference mitigation is discussed.

RFI mitigation workshop - RFI2010,

March 29-31, 2010

Groningen, the Netherlands

\footnotetext{
* Speaker.
} 


\section{Introduction}

Radio-astronomical observations with major instruments equipped with sensitive detectors always involve a number of interfering factors. Electromagnetic interference makes a considerable fraction of the operating frequency range of the RATAN-600 radio telescope inaccessible for radioastronomical studies. At the same time, the continuation of multifrequency operation of such a versatile instrument as RATAN-600 is of great importance for most astrophysical tasks. The use of the widest possible detection bands (up to several $\mathrm{GHz}$ ) in order to achieve the limiting sensitivity further complicates the problem. As is well known, in radio astronomy only narrow frequency bands - from several $\mathrm{MHz}$ to several hundred $\mathrm{MHz}$ - are protected against interference. The wellknown saying "per aspera ad astra" ("through the thorns to the stars") characterizes the situation with interference in radio-astronomical observations in the best possible way .

\section{The method of time-frequency interference mitigation}

The time-frequency method of interference mitigation is based on the following : 1)Analysis - the input band of the radiometer is subdivided into "narrow" frequency channels, with an independent detection section and rapid signal processing in each. 2)Synthesis - the wide radiometric band is synthesized by summing of the narrow channels, each of them cleaned from the short and large pulses with real-time digital signal processing. Block diagram of the "interference-mitigation adapter" of 13-cm radiometer is shown in Figure 1. The corresponding devices for the 31- and 49$\mathrm{cm}$ radiometers have a similar layout and differ only in the number of "narrow" channels (there are a total of four such adapters in the $31-\mathrm{cm}$ radiometer). This architecture allowed the interference situation to be studied at RATAN-600. These studies revealed a large fraction of pulsed interference at the time of measurements. The typical appearance of pulsed interference in all "narrow" channels of the 31-cm radiometer is shown in Figure 2. Shown are the individual half-periods of modulation in all four channels of the radiometer in the "antenna"-"dummy antenna" sequence for each channel. As is evident from the figure, interference is present only in the half-period of modulation, which corresponds to the sky observations ("antenna") and is absent in the half-period where the signal of the comparison channel (the "dummy antenna") is measured. This behavior is indicative of interference that is external with respect to the radiometer. The method of time-frequency mitigation at the level of signal processing consists of two stages. The first stage involves on-line removal in the digital signal processor of pulses whose duration does not exceed $80 \%$ of the halfperiod of modulation of the radiometer and whose amplitude exceeds the given threshold (e.g., $3 \sigma$ ). Signal counts in these intervals are substituted by the preceding "normal" count that met the $3 \sigma$ criterion. However, if the duration of interference exceeds the specified limit (on the order of 100 milliseconds), this interference is considered to be "long" so that its removal would break significantly the statistics of the channel and the corresponding interference is not removed. This determines the boundary of applicability of the method.

The second stage consists of off-line frequency interference mitigation. The central idea of the method consists in synthesizing the wide frequency band of the radiometer from the "narrow" channels during subsequent data reduction. If despite the first-stage reduction (mitigation of time- 
domain interference), some channels still contained long-duration interference, such channels were not included into the analysis.

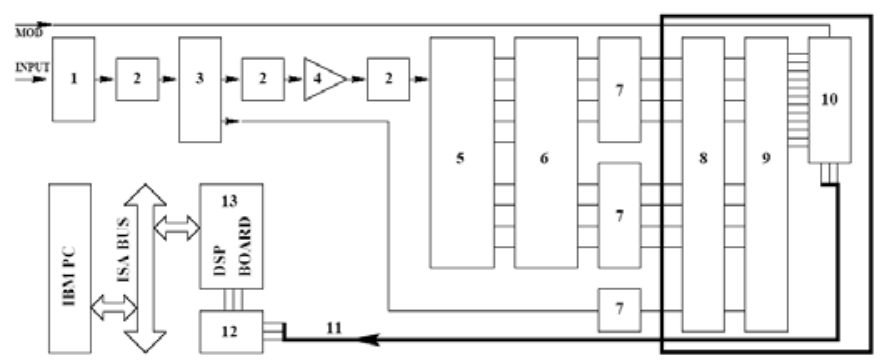

Figure 1: Block diagram of the anti-interference adapter to the 13 -cm radio radiometer. 1) $2.12-2.52 \mathrm{GHz}$ band microwave; 2) gates; 3) signal divider by 2 ; 4) amplifier(about $40 \mathrm{~dB}$ ) for compensating subsequent division of signal by 8 ; 5) signal divider by 8 ; 6) a bank of $50 \mathrm{MHz}$ wide microwave filters; 7) preliminary low-frequency amplifier with quadratic detectors at the input; 8) low-frequency filter to match the operating band of the analog-digital converter; 9) $\mathrm{ADC}(10-12$ bits) with a multiplexer at the input; 10) parallel-toserial code converter; 11) link to a digital signal processor on an ISA PC bus MOD-signal of the radiometer modulation; INPUT-input of the microwave signal.



Figure 2: The typical appearance of pulsed interference in all "narrow" channels of the 31-cm radiometer. The individual half-periods of modulation in all four channels of the radiometer in the "antenna"-"noise source" sequence are shown for each channel

The constraint of $80 \%$ half-period of the radiometer modulation (meander) on pulsed interference mitigation is an empirical rule, which meets the requirement that information of each halfperiod of modulation should participate in the output signal of the radiometer, i.e., that there would be no data gaps. This is due to the fact that all decameter-wave radiometers have a noise injection (RNI) scheme, where the sky signal (one half-period of the modulation) is compared to the signal of the internal noise source (during the second half-period) during each modulation period. Moreover, pulsed interference can be detected and eliminated only during one half-period because of the discontinuity of the signal at the boundary of half-periods. 
Figure 3 illustrates the application of the time-frequency method of interference mitigation to the $31-\mathrm{cm}$ radiometer. It should be pointed out that the 13 -and $49-\mathrm{cm}$ radiometers allowed data recording in the full radiometric bandwidth while switching off the online filtering of pulsed interference (record f). It is evident from this record, that during this observation the interference far exceeded the amplitude of the radio source. The pulsed interference has been successfully suppressed by filtering in the digital signal processor. As a result, all "narrow" channels became suitable for synthesizing the full bandwidth of the radiometer $(120 \mathrm{MHz})$, - (see frame e).

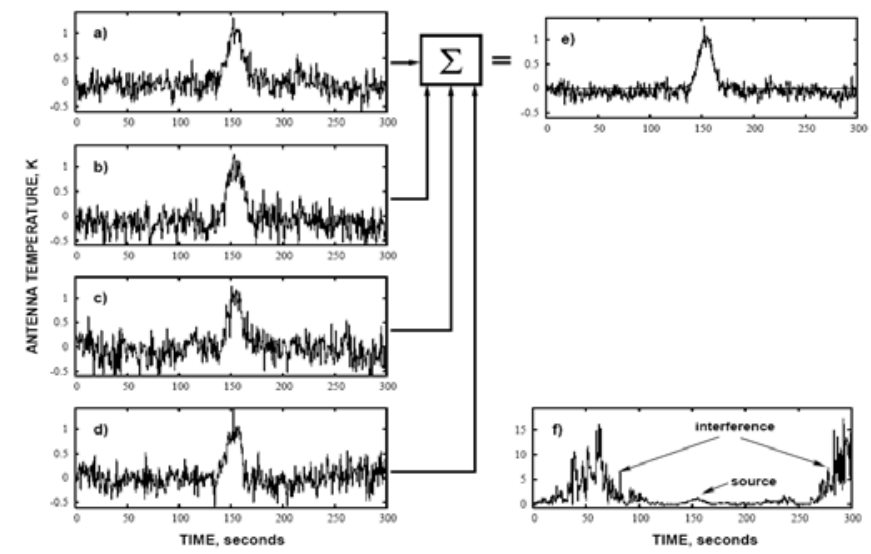

Figure 3: Illustration of the method of time-and-frequency mitigation of interference as applied to a 31-cm radio source using four "narrow" channels.

In the case of pulsed interference exclusively, the sequence of procedures "analysis $\rightarrow$ filtering $\rightarrow$ synthesis" allows reconstructing information in the full radiometric band of the radiometer albeit with a certain loss of sensitivity. Figure 4 demonstrates the efficiency of on-line filtering of pulsed interference. Observations with the $13-\mathrm{cm}$ radiometer were performed simultaneously with and without on-line filtering in the full band of the radiometer (400 MHz). The figure demonstrates virtually complete suppression of radar-station pulses in record (b). Residual "artifacts" indicate that the filtering algorithm operated at the limits of its capability when the duration of the pulse exceeded the permissible duration and the algorithm switched off for a short time.

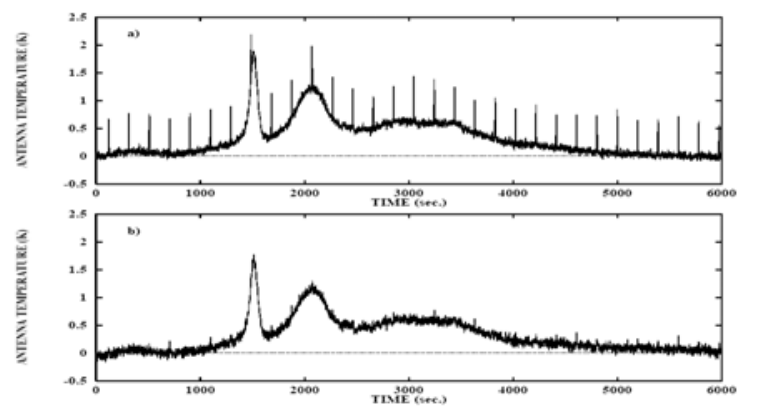

Figure 4: Illustration of on-line filtering of pulsed interference in a single band of the 13-cm radiometer during observations of a radio source: (a) without filtering and (b) with on-line filtering.

Figure 5 illustrates a case of observations with the $13-\mathrm{cm}$ radiometer at the limit of detector sensitivity in "narrow" channels. The (off-line) reduction of observations involved the synthesis of 
the full bandwidth of the radiometer frame (a) and the synthesis of the bandwidth after rejection of channel (e), frame (j). Interference is apparent only in channel (e), thereby preventing observation of the source in the full band of the radiometer. The source can be seen clearly in frame (j) and its parameters can be measured with high accuracy.

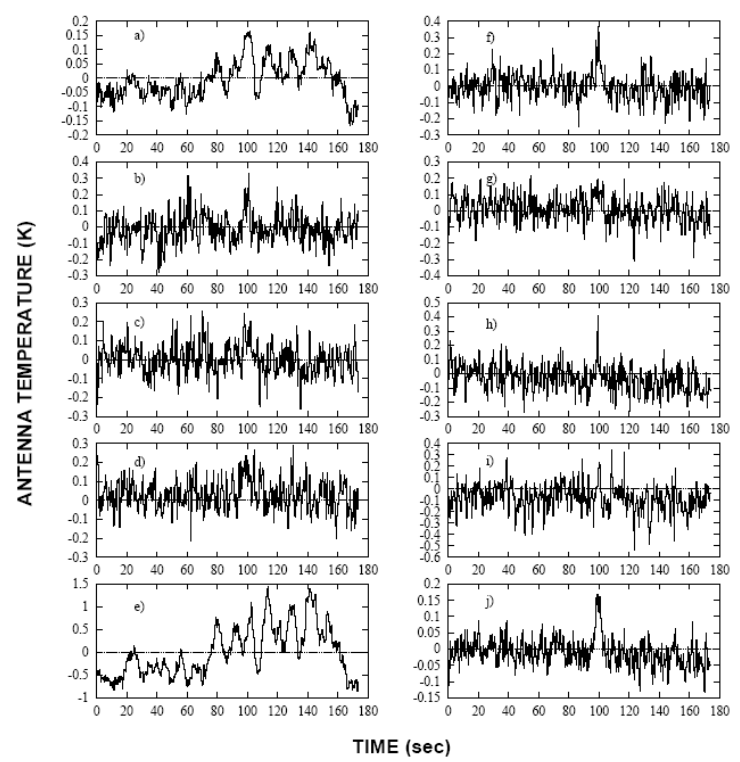

Figure 5: Record of the signal from radio source 1936+046 at the input of the 13-cm radiometer: (frame a) the signal of the "wideband" ( $400 \mathrm{MHz}$ ) channel without protection; (frame b-i) signals in "narrow" channels (each with a 50-MHz bandwidth) of the anti-interference system; (frame j) sum of the signals of the seven "narrow" channels uncontaminated by interference.

\section{Interference-monitoring capabilities at RATAN-600}

Two methods of studying the electromagnetic environment have been developed at RATAN600. In the first measurement system (Figure 6, left) the detector fully covers the operating decimeter range of RATAN-600. However, the sensitivity of this detector and a two-meter parabolic antenna, is much lower than that of the modern radiometer of the RATAN-600. For this reason, we acquired and installed an additional low-noise amplifier at the detector input $(+17 \mathrm{~dB})$ for precision measurements. This measurement system can be used for visual recording of the interference. We developed additional software to allow control of the measurement modes, recording of the detected interference to a personal computer (PC), and to receive measurement reports. The system runs under OS Linux and allows automatic scanning of the selected frequency band using different types of demodulation (PULSE, AM, FM, LOG) with the required frequency resolution and integration time. Figure 6 shows the block diagram of the measurement system, which is mounted at the upper edge of one of the secondary mirrors of the RATAN-600.

The second system to measure electromagnetic environment at the radio telescope site uses a spectrum analyzer (FSU-46; 0-46GHz) as a radiometer back-end (after the band pass filter) insert space (Figure 6, right). This system allows measurements on an absolute power scale $(\mathrm{dBm})$. 

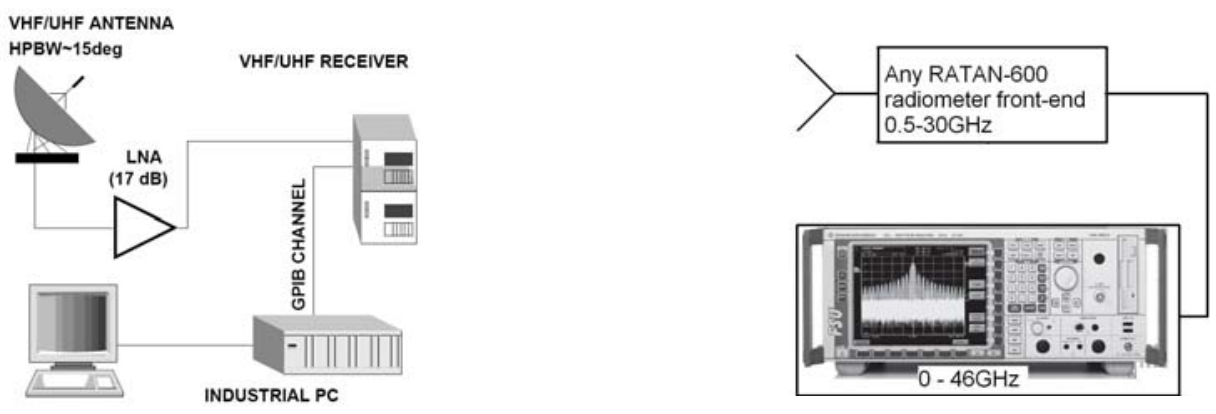

Figure 6: Block diagrams of interference-measurement setups at RATAN-600.

Figure 7 shows the measurement results of radio-frequency interference at RATAN-600 using the electromagnetic environment measuring setup.
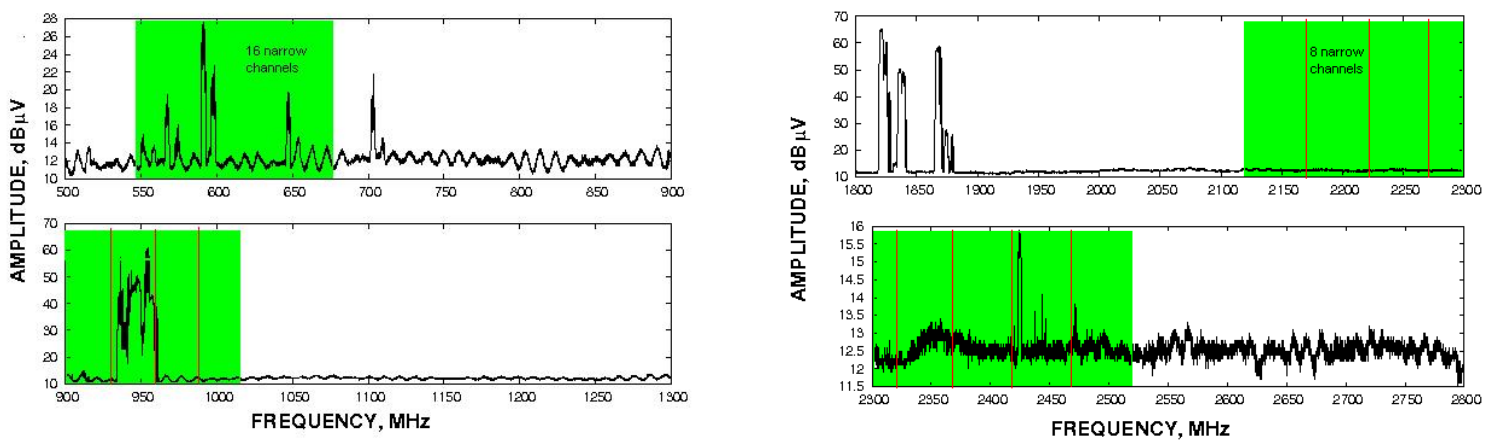

Figure 7: Radio-frequency emission detected at RATAN-600 using the electromagnetic environment measuring setup in the RATAN-600 frequency ranges (green zones): $546-677 \mathrm{MHz}(49 \mathrm{~cm}), 900-1020 \mathrm{MHz}(31$ $\mathrm{cm})$, and $2120-2520 \mathrm{MHz}(13 \mathrm{~cm})$. The red lines indicate the subdivided narrow channels

\section{Conclusions}

The complexity of the electromagnetic environment requires the use of modern hardware and software tools to successfully do interference mitigation in radio astronomy. The time-frequency approach described in this paper proved to be an efficient method to deal with the worst frequency ranges in radio astronomy. Further development of hardware and algorithms are required to obtain high quality radio-astronomical observations. In addition, new technical approaches need to be developed for interference mitigation at astronomical instruments, such as adaptive filtering.

\section{References}

[1] A.B. Berlin and P.A. Fridman. Real Time Radiometric Data Processing against Electromagnetic Pollution. In Proc. of the 25-th General Assembly of the International Union of Radio Science, Aug. 28-Sept.5, 1996, Lille, France (Lille, 1996, p.750)

[2] A.B. Berlin, E.V. Bulaenko, P.A. Fridman, RFI suppression device for RATAN-600 radiometer at $13 \mathrm{~cm}$, Problems of modern radio astronomy, v.3, 158-159(1997). 
[3] P. G. Tsybulev, A. B. Berlin, N. A. Nizhel'skij, M. G. Mingaliev, and D. V. Kratov, Interference-Mitigation Measures at RATAN-600 Radio Telescope, ISSN 1990-3413, Astrophysical Bulletin, 2007, Vol. 62, No. 2, pp. 193-201. Pleiades Publishing, Ltd., 2007 